\title{
Evaluación de la aceptabilidad de dos aceites vegetales con diferentes niveles de ácido alfa-linolénico en embarazadas de la Región Metropolitana de Chile
}

\author{
Acceptability assessment of two vegetable \\ oils with different level of alpha-linolenic \\ acid in pregnant women from the \\ Metropolitan Region of Chile
}

\begin{abstract}
Polyunsaturated Omega-3 fatty acids ( $\omega-3$ PUFA), particularly docosahexaenoic acid (C22:6 $\omega-3, D H A)$, are critical nutrients during pregnancy with a key role for visual and brain development. Fish, especially oily fish, is an excellent dietary source of DHA. However, its dietary consumption is low. Bioconversion from alpha-linolenic acid (C18: $3 \omega-3, A L A)$ to DHA is a limited metabolic pathway in humans, however, it increases during fertility and pregnancy. The chia seed oil (Salvia hispanica) is a good vegetal source of $\omega-3$ PUFA, containing a large proportion (60-65\% of total fatty acids) as ALA. The aim of this study was to compare the acceptability of two different commercial oils: chia oil $(63 \%$ ALA) and canola oil $(10 \%$ ALA) in a group of Chilean pregnant women. A sample of 41 women was included. Each participant performed: a) an assessment of the acceptability of two commercial oils(hedonic scale), b) a dietary intake questionnaire and, c) a test of knowledge related to $\omega-3$ PUFAs. Main results indicated that $57 \%$ of the participants properly recognized food sources of $\omega-3$ PUFA, and $12.2 \%$ met their dietary requirements. The characteristics that had the highest scores were the appearance of chia oil and the taste of canola oil. However, there were no significant differences between the overall acceptability of both evaluated oils. It is concluded that chia oil could be a new and useful alternative to increase dietary ALA intake in pregnant women.

Key words: pregnancy, w-3 fatty acids, alpha-linolenic acid, chia oil, vegetable oils, acceptability.
\end{abstract}

\section{INTRODUCCIÓN}

El embarazo se caracteriza por cambios fisiológicos que ocurren y son esenciales para promover y proteger el desarrollo del feto y también para preparar a la madre para el parto (1). Lo anterior determina un aumento de las necesidades nutricionales de macro y micronutrientes en relación al período pre-concepcional $(2,3)$. El crecimiento y desarrollo del feto dependen directamente de la alimentación de la madre, siendo el calcio, hierro, zinc, ácido fólico y los ácidos grasos (AG) esenciales nutrientes críticos en esta etapa (3). Entre estos últimos, el ácido linoleico (C18:2 $\omega-6, A L)$ y el ácido a-linolénico (C18:3
Alejandra Valencia C. $(1,2)$ Rodrigo Valenzuela B. (1) Karla Bascuñán G. (1) Rodrigo Chamorro (1) Cynthia Barrera R. (1) Mariana Faune P. (1) Macarena Jara N. (1) Cecilia Kuratomi M. (1) Andrea Moraga F. (1) Daniela Silva R. (1)

(1) Escuela de Nutrición y Dietética. Facultad de Medicina. Universidad de Chile. Santiago, Chile. (2) Departamento de Nutrición, Facultad de Medicina. Universidad de Chile. Santiago, Chile.

Dirigir la correspondencia a: Profesora Alejandra Valencia C. Escuela de Nutrición y Dietética y Departamento de Nutrición Facultad de Medicina. Universidad de Chile Casilla 1227. Independencia, Santiago, Chile Fono: 56-2-29786179 Fax: 56-2-29786182.

E-mail: avalencia@med.uchile.cl

Este trabajo fue recibido el 22 de Octubrede 2013 y aceptado para ser publicado el 25 de Enero de 2014.

$\omega-3, A L A)$ constituyen dos principales precursores metabólicos de las familias de AG. Considerando que el ser el humano no tiene la capacidad metabólica para incorporar dobles enlaces en las posiciones 3 y 6 de los AG (contando como carbono número 1 el carbono del grupo metilo, $\mathrm{CH} 3$ ), tanto el $\mathrm{AL}$ como el ALA se consideran AG esenciales y obliga a ingerirlos a través de la dieta (4). Respecto al ALA, este ácido graso es el precursor metabólico de los AG poliinsaturados de cadena larga (AGPICL) $\omega-3$, tales como el ácido eicosapentaenoico (C20:5 $\omega-3$, EPA) y el ácido docosahexaenoico (C22:6 $\omega-3$, $D H A)$. El AL es el precursor de los (AGPICL) $\omega-6$, tales como 
el ácido araquidónico (C20:4 $\omega-6$, AA) (4).

Durante el embarazo es esencial el aporte dietario de AGPICL $\omega-3$, especialmente de DHA, con el objetivo de favorecer un adecuado desarrollo del sistema nervioso y visual (3). La evidencia ha mostrado que la suplementación con AGPICL $\omega$-3 durante el embarazo y lactancia tiene beneficios a nivel de desarrollo cognitivo en recién nacidos y niños, pero no tendría un efecto en el desarrollo visual. Sin embargo, la suplementación de dosis de 100 mg de DHA más 200 mg de ácido araquidónico tendría implicancias en el desarrollo visual en recién nacidos de término (5). Al respecto, el requerimiento diario de ALA durante el embarazo es de 1,4 g/ día (6), y de acuerdo a FAO/OMS 2010 el requerimiento de EPA y DHA se ha establecido en un mínimo de $300 \mathrm{mg} /$ día en embarazadas y nodrizas, y de $200 \mathrm{mg}$ /día exclusivamente de DHA (7), el que debiese provenir principalmente de la dieta. Otras recomendaciones internacionales señalan que la ingesta debería ser entre 200 a 500 mg/día de $\operatorname{DHA}(8,9)$. De esta manera las recomendaciones nutricionales apuntan a aumentar el consumo de AGPICL $\omega$-3 mediante la ingesta de productos marinos, en especial pescados.

Las principales fuentes alimentarias de $A L$ son los aceites de maravilla (o girasol) (60\% AL), maíz (58\% AL) (6), mientras que el ALA se encuentra en los aceite de soja (7\% ALA), canola (11\% ALA), linaza (54\% ALA), chía (65\% ALA). A su vez, fuentes directas de AGPICL $\omega-3$ (EPA y DHA) se limitan casi exclusivamente a los pescados grasos, siendo el atún, la sardina, el jurel, el salmón y las anchoas los más importantes (10-12). Es necesario considerar que el contenido de EPA y DHA de los pescados varía considerablemente según la especie y contenido de grasa, ubicación geográfica y alimentación, observándose menores concentraciones en peces de cultivo que en aquellos de aguas profundas y frías (13).

Lamentablemente en los últimos años algunos estudios indican que los pescados grasos presentarían un alto contenido de componentes tóxicos como el metil-mercurio, especialmente el pez espada, caballa o blanquillo (14). El consumo de este tipo de pescados ha sido cuestionado durante el embarazo (15), sin embargo, está descrito que el consumo de $200 \mathrm{~g}$ de pescado a la semana, es seguro para la población general (1618) y corresponde a la recomendación que realiza el Estado de Chile en las guías alimentarias para la población chilena (19). No obstante lo anterior, el consumo de AGPICL $\omega-3$ es insuficiente en Chile. Datos nacionales indican que sólo $39.4 \%$ de la población consume pescado una o más veces por semana (20), lo que se estima un aporte de DHA del orden de 50 mg/día, muy por debajo de la recomendación (21). Ante esta situación, existen diferentes suplementos nutricionales con un alto contenido de EPA y DHA fabricados a partir de aceite de pescado, cuyas características organolépticas (sabor y olor), sumado a problemas de tolerancia digestiva (flatulencia aumentada con un desagradable sabor a pescado), produce un frecuente rechazo a este tipo de suplementos (12).

A nivel nacional se han implementado programas de suplementación y fortificación alimentaria durante el embarazo, destacando el programa de alimentación complementaria para el embarazo "Purita mamá." Este producto, que es una bebida láctea se entrega desde el año 2008 y contiene 90 mg DHA/200 mL (22). Sin embargo, en la Encuesta Nacional de Aceptabilidad de la "Bebida láctea purita mamá" se observó una baja aceptabilidad por parte de las mujeres beneficiarias (21). Frente a lo anterior, el desarrollo de nuevas alternativas alimentarias resulta fundamental para intentar cubrir los requerimientos de ALA, EPA y DHA en la población, particularmente en embarazadas y nodrizas.

La promoción del consumo de aceites vegetales que presentan un alto contenido de ALA (sobre el 50\%), podría ser una nueva e interesante estrategia y eventualmente a partir de estudios de conversión, impactar positivamente en los cambios en la concentración de EPA y DHA durante el embarazo y lactancia (23). El aceite de la semilla de chía (Salvia hispánica) contiene entre un 60-65\% de ALA y adecuada estabilidad química, por su alto contenido de antioxidantes naturales (tocoferoles) (24), características que lo constituyen en una importante fuente dietaria de ALA. El presente estudio evaluó la aceptabilidad de dos aceite vegetales (chía y canola) con diferentes niveles de ALA, en un grupo de embarazadas de la Región Metropolitana de Santiago de Chile.

\section{SUJETOS Y MÉTODOS Sujetos}

El estudió se efectuó en 41 embarazadas de 17 y 43 años de edad (promedio $30 \pm 6.31$ años) residentes en la Región Metropolitana de Santiago de Chile, que recibían atención regular en los Centros de Salud Familiar de las comunas de Independencia, Peñalolén, Santiago y de un consultorio en la provincia de Melipilla. Las evaluaciones se realizaron desde septiembre a octubre de 2012. Este estudio fue aprobado por el comité de Ética de la Facultad de Medicina, Universidad de Chile. Todas las participantes firmaron autorización escrita mediante un consentimiento informado. El cálculo del tamaño muestral se determinó considerando como variable crítica el cambio en la aceptabilidad del aceite de chía respecto al aceite de canola (variación inferior al 30\%), con una significancia estadística a nivel de $\mathrm{p}<0,05$, con un poder de 0.9 y un margen de pérdida de sujetos del $20 \%$. Utilizando el software n-Query Advisor, considerando el modelo experimental y tipo de prueba estadística a realizar.

Evaluación del conocimiento respecto a los AGPI $\omega-3$

Para determinar el nivel de conocimientos de cada participante en relación a las características generales e importancia de los AGPI $\omega-3$ para la salud, se utilizó una encuesta de conocimientos generales. Esta consistió en preguntas relacionadas con su conocimiento sobre los AGPI $\omega-3$, características, beneficios y fuentes dietarias. Igualmente, se preguntó por su conocimiento del aceite de semilla de chía. La encuesta de conocimientos generales a utilizar fue revisada por tres nutricionistas expertas en ese tipo de encuestas. La encuesta previo a su aplicación fue validada en un grupo de 8 mujeres embarazadas.

\section{Aceite de chía y canola}

Los aceites vegetales utilizados en este estudio fueron un aceite de Canola (canadian oil low erucic acid) y de chía (salvia hispánica). En relación a su perfil de AG, el aceite de semilla de chía presentó un 78-80 \% de AGPI, 7-8 \% de AG monoinsaturados (AGMI), y entre un 10-11\% de AG saturados (AGS). En relación a los AGPI, entre un 60 y $65 \%$ de estos fueron ALA. Por su parte, el aceite de canola presentó un 30$28 \%$ de AGPI, 58-60 \% AGMI, y entre un 8-12 \% de AGS. Dentro de los AGPI, entre un 9-11\% de estos fueron ALA. Ambos aceites presentaron color y viscosidad característica de los aceites vegetales.

\section{Evaluación del consumo de AGPI $\omega-3$}

Para determinar la ingesta dietaria promedio diaria, con especial énfasis en las fuentes de AGPI $\omega-3$, a cada participante 
se le aplicó dos instrumentos, un recordatorio de 24 horas (R24-H) y una encuesta de tendencia de consumo modificada (ETCM); en esta última, se preguntó por la frecuencia y cantidad del consumo de alimentos fuentes de AGPI $\omega-3$ durante el último mes. Para estimar la ingesta diaria promedio de AGPI $\omega-3$, los datos de consumo alimentario obtenidos a partir del R24-H y la ETCM fueron analizados usando el programa Food Processor SQL (ESHA Research, Salem, OR).

\section{Evaluación de la aceptabilidad del} aceite de chía y canola

Para realizar una evaluación sensorial de ambos aceites, se conformó un panel sensorial para evaluar el aceite de chía y de canola. Para la evaluación cada participante recibió una muestra de cada uno de los aceites en un trozo de pan. Luego los investigadores entregaron instrucciones para que las embarazadas evaluaran cada una de las características de los aceites indicando su nivel de agrado, marcando con una $X$ el punto en la escala que mejor describía su sentir. Las mujeres evaluaron cinco características del producto: sabor, olor, color, consistencia y apariencia; además se les solicitó evaluar la aceptabilidad general, a través de una escala hedónica de 1 a 5 puntos que consideró las categorías: me disgusta mucho, me disgusta, no me gusta ni me disgusta, me gusta y me gusta mucho.

Análisis estadístico

Todos los análisis estadísticos se realizaron utilizando el programa SPSS $®$ v.11.5 (SPSS Inc., Chicago, Illinois). Para determinar si existía una asociación entre el consumo de AGPI $\omega$-3 y el conocimiento que las participantes tenían con respecto a estos AG se utilizó la prueba de correlación de Spearman. Para evaluar diferencias entre la aceptabilidad del aceite de chía y de canola se utilizó la prueba de Wilcoxon. Para todas las pruebas se aceptó como significativo un valor $p<0,05$.

\section{RESULTADOS}

Caracterización de la muestra

La muestra se conformó por 41 embarazadas, de las cuales un $34.1 \%$ había completado la enseñanza media, un $65 \%$ de las participantes se encontraba en su tercer trimestre de embarazo y la mayoría de ellas tenía un estado nutricional pre-gestacional normal (56.7\%). (tabla 1).

\section{Encuesta de conocimiento}

En la tabla 2 se indica el porcentaje de respuestas correctas de la prueba de conocimiento aplicadas a las embarazadas. En el ítem I, sólo 16,6\% del total de la muestra manifestó conocer el aceite de chía, mientras que la misma pregunta relacionada a los AGPI $\omega-3$ el 71,4\% respondió afirmativamente. En el ítem III, la primera pregunta referida a ¿qué son los AGPI $\omega-3$ ? sólo 14,2\% respondió en forma correcta. Con respecto a los beneficios que tiene el consumo de AGPI $\omega-3$ para la salud, el $52 \%$ de la muestra aseguró conocerlos. Finalmente, un $57 \%$ de las embarazadas reconoció correctamente los alimentos fuentes de AGPI $\omega-3$.

Estimación del consumo de ALA

La ingesta promedio de AGPI $\omega-3$ a partir de los alimen-

TABLA 1

Caracterización de la muestra

\begin{tabular}{|c|c|c|c|c|c|}
\hline Nivel educacional & $\begin{array}{l}\text { Porcentaje } \\
\text { (\%) }\end{array}$ & $\begin{array}{l}\text { Periodo } \\
\text { gestacional }\end{array}$ & $\begin{array}{c}\text { Porcentaje } \\
\text { (\%) }\end{array}$ & $\begin{array}{l}\text { Estado nutricional } \\
\text { (pre - gestacional) }\end{array}$ & $\begin{array}{c}\text { Porcentaje } \\
(\%)\end{array}$ \\
\hline Básica & $4(9,7 \%)$ & $1^{\circ}$ trimestre & $1(2,5 \%)$ & Bajo peso & $1(2,7 \%)$ \\
\hline Enseñanza media incompleta & $4(9,7 \%)$ & $2^{\circ}$ trimestre & $13(32,5 \%)$ & Eutrófico & $21(56,7 \%)$ \\
\hline Enseñanza media completa & $14(34,1 \%)$ & $3^{\circ}$ trimestre & $26(65 \%)$ & Sobrepeso & $8(21,6 \%)$ \\
\hline Técnico incompleta & $1(2,4 \%)$ & & & Obesidad & $7(18,9 \%)$ \\
\hline Técnico completa & $7(17,0 \%)$ & & & & \\
\hline Universitaria incompleta & $4(9,7 \%)$ & & & & \\
\hline Universitaria completa & $7(17,0 \%)$ & & & & \\
\hline Total $(n)$ & 41 & & 40 & & 37 \\
\hline
\end{tabular}

TABLA 2

Porcentajes de respuestas correctas de la encuesta de conocimientos.

Pregunta

¿Conoce el aceite de Chía?

¿Conoce los ácidos AGPI $\omega-3$ ?

¿Qué son los AGPI $\omega-3$ ?

¿Qué beneficios para la salud tiene el consumo de AGPI $\omega-3$ ?

¿En qué alimentos se encuentran los AGPI $\omega-3$ ?
Respuesta afirmativa y alternativa correcta

$16,6 \%$

$71,4 \%$

$14,2 \%$

$52 \%$

$57 \%$ 
tos fuentes de ellos, determinado a través del análisis de ETCM fue de $1.33 \pm 2.51 \mathrm{~g} /$ día. Sólo un $12.2 \%$ de la muestra cumplió con el requerimiento diario de ALA, consumiendo al menos $1.4 \mathrm{~g} /$ día. No se observó una correlación entre la ingesta diaria promedio de AGPI $\omega-3$ y el conocimiento que las participantes del estudio tenían acerca de ellos $(r=0.19, p=0.23)$.

Aceptabilidad de aceites de chía y canola

A partir de la escala hedónica utilizada para medir la aceptabilidad de ambos aceites, se observaron distintos puntajes de aceptación y rechazo para las diversas características organolépticas. Las características que menos gustaron a las embarazadas fueron el olor y el sabor del aceite de chía, mientras que para el aceite de canola fue el color, la apariencia y la consistencia. Por el contrario, las características organolépticas que presentaron los mayores puntajes fueron la apariencia del aceite de chía, y el sabor del aceite de canola. Sin embargo, no hubo diferencias significativas ( $p>0,05$ para todas las comparaciones) al evaluar las características organolépticas según el tipo de aceite testeado.

\section{DISCUSIÓN}

A partir de los resultados obtenidos se comprueba que los aceites de canola y chía evaluados no difirieron entre sí con respecto a sus características organolépticas (sabor, olor, color, consistencia y apariencia, además de aceptabilidad en general). Lo anterior sustenta el considerar el uso de una semilla como la chía, para aumentar la ingesta de AGPI $\omega-3$ en un grupo de la población cuyo requerimiento de AGPI- $\omega$ debe ser cuidadosamente cubierto. Un aspecto a destacar es que a pesar de que no se observó diferencias significativas respecto a la aceptabilidad entre los dos tipos de aceites, si se considera solo el olor como parámetro evaluado, el aceite de chía obtuvo un menor puntaje en relación al aceite de canola (3 y 4 respectivamente), probablemente, el mayor porcentaje de ALA que presenta el aceite de Chía podría ser una razón para lo anterior. Por otro parte, en relación al parámetro apariencia, cabe mencionar que el aceite de chía tuvo una buena aceptabilidad lo que puede ser relevante, ya que se recomienda consumirlo crudo por la inestabilidad al calor de los AGPI $\omega-3$.

Con respecto a la ingesta del AGPI $\omega$-3, especialmente de ALA, solamente un porcentaje menor (12.2\% de la muestra) cumplió con sus requerimientos diarios. En las mujeres que cumplieron el requerimiento de AGPI $\omega-3$ presentaban mayor consumo de Leche Purita Mamá y pescado. Sin embargo, algunas de ellas señalaron consumir el pescado frito, perdiendo y/o disminuyendo las propiedades de los AGPI $\omega-3$. Por lo tanto, la ingesta promedio diaria de AGPI $\omega-3$ del $87.8 \%$ de las mujeres que participaron este estudio probablemente sea insuficiente. Estos datos son similares a los que se describen en la última Encuesta Nacional de Salud 2009-2010 (20). Por otra parte, se hace necesario sensibilizar al grupo de embarazadas en relación a los AGPI $\omega-3$ y a los beneficios que tienen durante los meses de gestación y lactancia materna, debido a que en este estudio quedó evidenciado que más del $50 \%$ de las mujeres no conoce los AGPI $\omega-3$. Es importante destacar que el consumo de ALA a partir del aceite de chía, aceite que tuvo una buena aceptabilidad- podría representar una medida dietética factible de implementar para cumplir con los requerimientos de este tipo de AG en este grupo etario. Este aceite se caracteriza por su alta cantidad de ALA (25), lo que lo convierte una buena alternativa en ese sentido. Considerando las características de este aceite resulta importante evaluar el aporte de ALA presente en el aceite de chía sobre la capacidad de bioconversión metabólica de los AGPICL $\omega-3$, especialmente a DHA, en embarazadas chilenas.

\section{CONCLUSIÓN}

La aceptabilidad del aceite de chía en términos de sus características organolépticas es comparable al de un aceite de canola, ambas buenas fuentes vegetales de ALA. El consumo de aceite de chía durante el embarazo y el período de lactancia podría contribuir en entregar los beneficios nutricionales del ALA en el feto y la madre, y se convertiría en una alternativa para aumentar su ingesta dietaria. Estudios que evalúen la utilización de este aceite y posibles modificaciones de marcadores fisiológicos de estatus de ALA y sus productos finales resultan necesarios para evaluar sus efectos metabólicos durante el embarazo y lactancia.

\section{RESUMEN}

Los ácidos grasos (AG) poliinsaturados omega 3 (AGPI $\omega-3), y$ especialmente el ácido docosahexaenoico (C22:6 $\omega-3, D H A)$ participan en el desarrollo cerebral y visual del feto y del lactante, constituyendo nutrientes críticos durante el embarazo. Los pescados grasos, representan una excelente fuente de consumo de DHA, sin embargo, su consumo a nivel poblacional es bajo. El ácido alfa-linolénico (C18:3 $\omega-3$, ALA) es el precursor del DHA, vía metabólica limitada en el ser humano, pero que aumenta en mujeres en edad fértil y embarazadas. El aceite de semilla de chía (Salvia hispánica) contiene en una gran cantidad de AGPI $\omega-3$ (60-65\% del total de AG) y su aceite podría representar una nueva fuente de este tipo de AG. El objetivo de este estudio fue comparar la aceptabilidad de un aceite de canola (10\% ALA) y chía $(63 \%$

\section{TABLA 3}

Encuesta aceptabilidad del aceite de chía y canola.

\section{Característica evaluada}

Sabor

Olor

Color

Consistencia

Apariencia general
Aceite de chía*

4

3

4

4

4
Aceite de canola*

4

4

3

4

4

\footnotetext{
* Mediana para cada uno de los aceites evaluados. No hubo diferencias significativas entre los aceites $p>0,05$.
} 
ALA) en embarazadas chilenas. Se estudió una muestra de 41 embarazadas quienes respondieron una encuesta de aceptabilidad (escala hedónica), una encuesta de tendencia de consumo modificada y una prueba de conocimiento respecto a los AGPI $\omega-3$. Los resultados indicaron que el $57 \%$ de las embarazadas reconoció correctamente los alimentos fuentes de AGPI $\omega-3$ y $12.2 \%$ de ellas cumplió con sus requerimientos dietarios. Las características que presentaron los mayores puntajes fueron la apariencia del aceite de chía, y el sabor del aceite de canola. Sin embargo, no existió diferencia significativa entre la aceptabilidad de ambos aceites evaluados. Se concluye que el aceite de chía podría ser utilizado como una alternativa dietaria de buena fuente de ALA en embarazadas.

Palabras clave: AG $\omega-3$, embarazadas, ácido alfa-linolénico, aceite de chía, aceptabilidad.

\section{BIBLIOGRAFÍA}

1. Carlin A, Alfirevic Z. Physiological changes of pregnancy and monitoring. Best Practice \& Research Clinical Obstetrics \& Gynaecology. 2008;22:801-23.

2. Díaz $A C L$, Valeria $V O H$, Biolley $H E E$. Ingesta dietaria de nutrientes críticos en embarazadas. Rev Chil Nutr. 2005;32:225-31.

3. Valenzuela $B$ A, Nieto $K S$. Ácidos grasos omega-6 y omega-3 en la nutrición perinatal: su importancia en el desarrollo del sistema nervioso y visual. Rev Chil Ped. 2003;74:149-57.

4. Brenner RR, Peluffo RO. Regulation of unsaturated fatty acids biosynthesis. I. Effect of unsaturated fatty acid of 18 carbons on the microsomal desaturation of linoleic acid into gamma-linolenic acid. Biochim Biophys Acta. 1969;176:471-9.

5. Eilander A, Hundscheid DC, Osendarp SJ, Transler C, Zock $P L$. Effects of $n-3$ long chain polyunsaturated fatty acid supplementation on visual and cognitive development throughout childhood: a review of human studies. Prostaglandins Leukot Essent Fatty Acids. 2007;76:189-203.

6. Dietary Reference Intakes for Energy, Carbohydrate, Fiber, Fat, Fatty Acids, Cholesterol, Protein, and Amino Acids (Macronutrients). The National Academies Press; 2005.

7. Fats and fatty acids in human nutrition: Report an expert consultation. FAO Food and Nutrition Paper Nº 91; 2010.

8. Koletzko B, Lien E, Agostoni C, Bohles H, Campoy C, Cetin $l$, et al. The roles of long-chain polyunsaturated fatty acids in pregnancy, lactation and infancy: review of current knowledge and consensus recommendations. J Perinat Med. 2008;36:5-14.

9. Calder PC, Dangour AD, Diekman C, Eilander A, Koletzko $B$, Meijer GW, et al. Essential fats for future health. Proceedings of the 9th Unilever Nutrition Symposium, 26-27 May 2010. Eur J Clin Nutr. 2010;64:S1-13.

10. Morales PJ, Valenzuela B R, González M D, González E M, Tapia O G, Sanhueza $C$ J, et al. Nuevas fuentes dietarias de acido alfa-linolénico: una visión crítica. Rev Chil Nutr. 2012;39:79-87.
11. Sanhueza J, Nieto S, Valenzuela A. Ácido docosahexaenoico (DHA), desarrollo cerebral, memoria aprendizaje: La importancia de la suplementación perinatal. Rev Chil Nutr. 2004:31:84-92.

12. Valenzuela B A, Sanhueza C J. Aceites de origen marino; su importancia en la nutrición y en la ciencia de los alimentos. Rev Chil Nutr. 2009;36:246-57.

13. Valenzuela B A, Sanhueza C J, de la Barra D F. El aceite de pescado: Ayer un desecho industrial, hoy un producto de alto valor nutricional. Rev Chil Nutr. 2012;39:201-9.

14. Lando AM, Fein SB, Choiniere CJ. Awareness of methylmercury in fish and fish consumption among pregnant and postpartum women and women of childbearing age in the United States. Environ Res. 2012;116:85-92.

15. Murata $K$, Yoshida M, Sakamoto M, Iwai-Shimada M, Yaginuma-Sakurai $K$, Tatsuta $N$, et al. Recent evidence from epidemiological studies on methylmercury toxicity. Nihon Eiseigaku Zasshi. 2011;66:682-95.

16. Golding J, Steer CD, Hibbeln JR, Emmett PM, Lowery $T$, Jones R. Dietary Predictors of Maternal Prenatal Blood Mercury Levels in the ALSPAC Birth Cohort Study. Environ Health Perspect. 2013;121:1214-8.

17. Traynor S, Kearney G, Olson D, Hilliard A, Palcic J, Pawlowicz $M$. Fish consumption patterns and mercury exposure levels among women of childbearing age in Duval County, Florida. J Environ Health. 2013;75:8-15.

18. Llorente Ballesteros MT, Vírseda Chamorro I, Peral Jiménez $R$, Sanz Grimaldi MJ, Ruiz Tejada MA, López Colón JL. Metilmercurio en el cabello de población infantil. Sanidad Militar. 2011;67:299-303.

19. Informe final "Estudio para revisión y actualización de las guías alimentarias para la población chilena". Ministerios de Salud. Gobierno de Chile. 2013.

20. Encuesta Nacional de Salud (ENS) Chile 2009-2010. Ministerio de Salud, Gobierno de Chile.

21. Contreras $M A$, Herrera $Y$, Rodríguez $O L$, Pizarro $Q T$, Atalah S E. Aceptabilidad y consumo de una bebida láctea con omega-3 en embarazadas y nodrizas del Programa Nacional de Alimentación Complementaria. . Rev Chil Nutr. 2011;38:313-20.

22. Castillo D C, Balboa C P, Raimann TX. Modificaciones a la Leche del Programa Nacional de Alimentación Complementaria (PNAC) en Chile. 2009. Rev Chil Ped. 2009;80:508-12.

23. Plourde M, Cunnane SC. Extremely limited synthesis of long chain polyunsaturates in adults: implications for their dietary essentiality and use as supplements. Appl Physiol Nutr Metab. 2007;32:619-34.

24. Ayerza R, Jr., Coates W. Effect of dietary alpha-linolenic fatty acid derived from chia when fed as ground seed, whole seed and oil on lipid content and fatty acid composition of rat plasma. Ann Nutr Metab. 2007;51:27-34.

25. Valenzuela $B R$, Tapia $O G$, González E M, Valenzuela $B$ A. Ácido grasos omega-3 (EPA Y DHA) y su aplicación en diversas situaciones clínicas. Rev Chil Nutr. 2011;38:356-67. 